\title{
ATP Hydrolysis
}

National Cancer Institute

\section{Source}

National Cancer Institute. ATP Hydrolysis. NCI Thesaurus. Code C19939.

ATP Hydrolysis is the cleavage of a high-energy phosphate bond of adenosine triphosphate by the addition of water. The energy derived from hydrolysis of ATP is used to drive metabolic reactions including nucleic acid and protein synthesis, to move molecules against concentration gradients (active transport), and to produce mechanical motion (contraction of microfibrils and microtubules). 